\title{
Two-Dimensional Superfluid Density in an Alkali Metal-Organic Solvent Intercalated Iron Selenide Superconductor $\mathrm{Li}\left(\mathrm{C}_{5} \mathrm{H}_{5} \mathrm{~N}\right)_{0.2} \mathrm{Fe}_{2} \mathrm{Se}_{2}$
}

\author{
P. K. Biswas, ${ }^{1, *}$ A. Krzton-Maziopa, ${ }^{2,3}$ R. Khasanov, ${ }^{1}$ H. Luetkens, ${ }^{1}$ E. Pomjakushina, ${ }^{2}$ K. Conder, ${ }^{2}$ and A. Amato ${ }^{1}$ \\ ${ }^{1}$ Laboratory for Muon Spin Spectroscopy, Paul Scherrer Institute, CH-5232 Villigen PSI, Switzerland \\ ${ }^{2}$ Laboratory for Developments and Methods, Paul Scherrer Institute, CH-5232 Villigen PSI, Switzerland \\ ${ }^{3}$ Faculty of Chemistry, Warsaw University of Technology, Noakowskiego 3, 00-664 Warsaw, Poland \\ (Received 21 September 2012; revised manuscript received 30 December 2012; published 28 March 2013; \\ publisher error corrected 5 April 2013)
}

\begin{abstract}
We report the low-temperature electronic and magnetic properties of the alkali metal-organic solvent intercalated iron selenide superconductor $\mathrm{Li}\left(\mathrm{C}_{5} \mathrm{H}_{5} \mathrm{~N}\right)_{0.2} \mathrm{Fe}_{2} \mathrm{Se}_{2}$ using muon-spin-spectroscopy measurements. The zero-field muon spin relaxation $(\mu \mathrm{SR})$ results indicate that nearly half of the sample is magnetically ordered and spatially phase separated from the superconducting region. The transverse-field $\mu \mathrm{SR}$ results reveal that the superfluid density of $\mathrm{Li}\left(\mathrm{C}_{5} \mathrm{H}_{5} \mathrm{~N}\right)_{0.2} \mathrm{Fe}_{2} \mathrm{Se}_{2}$ is two dimensional in nature. The temperature dependence of the penetration depth $\lambda(T)$ can be explained using a two-gap $s$-wave model. This implies that, despite the 2D nature of the superfluid density, the symmetry of the superconducting gap remains unaltered to the parent compound FeSe.
\end{abstract}

DOI: 10.1103/PhysRevLett.110.137003

PACS numbers: 74.70.Xa, 74.25.Ha, 76.75.+i

Since the discovery of the iron-based superconductor $\mathrm{LaFeAsO}_{1-x} \mathrm{~F}_{x}$ in 2008 [1], it has been fascinating to observe how the research in superconductivity has evolved during the last four years. In general, there are two classes of iron-based superconductors, chalcogenides and pnictides. Initially, the iron pnictide system received most of the attention of the scientific community due to its higher $T_{c}$ values, with a highest $T_{c}$ of $56 \mathrm{~K}$ for $\mathrm{Gd}_{1-x} \mathrm{Th}_{x} \mathrm{FeAsO}$ [2]. Recently, the iron chalcogenide system has generated much interest to the condensed matter physicists due to the development of new superconductors with increasing $T_{c}$ values and its coexistence and competition with strongly magnetic phases [3-9]. The parent compound of this system is FeSe, superconducting with a $T_{c}$ of $8.0 \mathrm{~K}$ at ambient pressure [10] and $37 \mathrm{~K}$ at $7 \mathrm{GPa}$ [11]. The $T_{c}$ value can be raised as high as $65 \mathrm{~K}$ for a single layered FeSe film grown on a $\mathrm{SrTiO}_{3}$ substrate [12-14]. The substitution of tellurium on the selenium site also increases $T_{c}$ to a maximum of $14.5 \mathrm{~K}$ at ambient pressure [15,16] and $23.3 \mathrm{~K}$ at $3 \mathrm{GPa}$ [17]. A further milestone in the evolution of iron chalcogenide superconductors has been achieved by intercalating alkali metals $(\mathrm{K}, \mathrm{Cs}, \mathrm{Rb})$ between FeSe layers which increases the $T_{c}$ values above $30 \mathrm{~K}[3,4,18]$. These alkali intercalated materials also have a second superconducting phase beyond $12 \mathrm{GPa}$ with a $T_{c}$ of $48 \mathrm{~K}$ [19]. Interest has been redoubled in this class of superconductors with the recent discovery of the enhancement of $T_{c}$ of FeSe above $40 \mathrm{~K}$ by the intercalation of a molecular spacer layer [20-23]. It has been claimed that the value of $T_{c}$ increases with an increasing interlayer distance between the FeSe layers [24]. Therefore, it is interesting to elucidate the nature of the superfluid density, pairing mechanism, and gap symmetry and how they evolve with the increasing interlayer distances in these intercalated FeSe superconductors.
In this Letter, we report on a detailed investigation of the superfluid density $\left(n_{s}\right)$ and the symmetry of the superconducting gap in the alkali metal-organic solvent intercalated iron selenide superconductor $\mathrm{Li}\left(\mathrm{C}_{5} \mathrm{H}_{5} \mathrm{~N}\right)_{0.2} \mathrm{Fe}_{2} \mathrm{Se}_{2}$ by the muon spin rotation or relaxation $(\mu \mathrm{SR})$ technique. Our results clearly demonstrate that nearly half of the volume fraction of $\mathrm{Li}\left(\mathrm{C}_{5} \mathrm{H}_{5} \mathrm{~N}\right)_{0.2} \mathrm{Fe}_{2} \mathrm{Se}_{2}$ is in a magnetically ordered state. The other half is paramagnetic and becomes fully superconducting below $T_{c}$. The observed $n_{s} \approx \lambda^{-2}(T)$ is found to be well described by a similar two-gap $s$-wave model, as seen in the parent compound $\mathrm{FeSe}_{0.85}$ [25]. Comparing these results with a related superconductor $\mathrm{Li}_{0.6}\left(\mathrm{NH}_{2}\right)_{0.2}\left(\mathrm{NH}_{3}\right)_{0.8} \mathrm{Fe}_{2} \mathrm{Se}_{2}$ [21], we found that the penetration depth increases with increasing FeSe layer separation. Our results strongly suggest that the 2D FeSe layer generates superconductivity in this class of materials and the value of $T_{c}$ depends only on the superfluid density within this FeSe layer.

$\mu \mathrm{SR}$ measurements were performed using the general purpose surface muon instrument located on the $\pi \mathrm{M} 3.2$ beam line of the Swiss Muon Source at the Paul Scherrer Institute, Villigen, Switzerland. Data were collected both in zero field (ZF) and transverse field (TF) modes. In the TF mode, an external magnetic field $(H=30 \mathrm{Oe})$ was applied perpendicular to the initial direction of the muon spin polarization. The magnetic field was applied above the superconducting transition temperature and the sample then cooled to base temperature, i.e., the so-called field-cooled procedure. A continuous-flow ${ }^{4} \mathrm{He}$ cryostat was used to collect the data between 1.6 and $250 \mathrm{~K}$. The number of positron events were 18 million for each data point.

A polycrystalline sample of $\mathrm{Li}\left(\mathrm{C}_{5} \mathrm{H}_{5} \mathrm{~N}\right)_{0.2} \mathrm{Fe}_{2} \mathrm{Se}_{2}$ was prepared via room temperature intercalation into the iron 
selenide matrix in a pyridine solution of the Li metal as described in Ref. [23]. A disk shaped pellet of the powder sample of $\mathrm{Li}\left(\mathrm{C}_{5} \mathrm{H}_{5} \mathrm{~N}\right)_{0.2} \mathrm{Fe}_{2} \mathrm{Se}_{2}$ with an $8 \mathrm{~mm}$ diameter and $2 \mathrm{~mm}$ thickness was mounted on a fork shaped sample holder. The pellet was covered with a thin layer of polymer and kept in an inert atmosphere to inhibit air decomposition.

Temperature dependence of the ac-magnetic susceptibility of $\mathrm{Li}\left(\mathrm{C}_{5} \mathrm{H}_{5} \mathrm{~N}\right)_{0.2} \mathrm{Fe}_{2} \mathrm{Se}_{2}$ was performed in a Quantum Design Physical Property Measurement System magnetometer under a magnetic field of $H_{\mathrm{ac}}=1 \mathrm{Oe}$, using a zero-field-cooled protocol. The inset of Fig. 1 shows a diamagnetic transition in the susceptibility data with a $T_{c}$ onset at 40(1) K. A rather large positive signal in the normal state susceptibility data shows the presence of a sizeable amount of ferromagnetic impurity in the sample. This may be due to the presence of some small clusters of Fe ions and other Fe-based magnetic phases such as $\alpha-$ $\mathrm{FeSe}, \mathrm{Fe}_{7} \mathrm{Se}_{8}, \mathrm{Fe}_{3} \mathrm{O}_{4}$ in the sample. Comparing the normal state susceptibility value with the saturation magnetization of elemental $\mathrm{Fe}, \mathrm{Fe}_{7} \mathrm{Se}_{8}$, and $\mathrm{Fe}_{3} \mathrm{O}_{4}$ we estimate that the amount of this magnetic impurity is not more than $1 \%$ of the sample mass. A similar scenario has also been observed in many other Fe-based superconductors [21,23,25-29].

In order to search for any magnetic anomalies (static or fluctuating) in $\mathrm{Li}\left(\mathrm{C}_{5} \mathrm{H}_{5} \mathrm{~N}\right)_{0.2} \mathrm{Fe}_{2} \mathrm{Se}_{2}$, we have performed a $\mathrm{ZF}-\mu \mathrm{SR}$ study. Figure 1 shows the ZF- $\mu \mathrm{SR}$ signals collected at 1.6, 50, and $250 \mathrm{~K}$ for $\mathrm{Li}\left(\mathrm{C}_{5} \mathrm{H}_{5} \mathrm{~N}\right)_{0.2} \mathrm{Fe}_{2} \mathrm{Se}_{2}$. The signals are almost identical and there is no precessional signal and no big change in the observed relaxation rate between data collected above and below $T_{c}$. However, we have observed a large drop of the asymmetry value of the

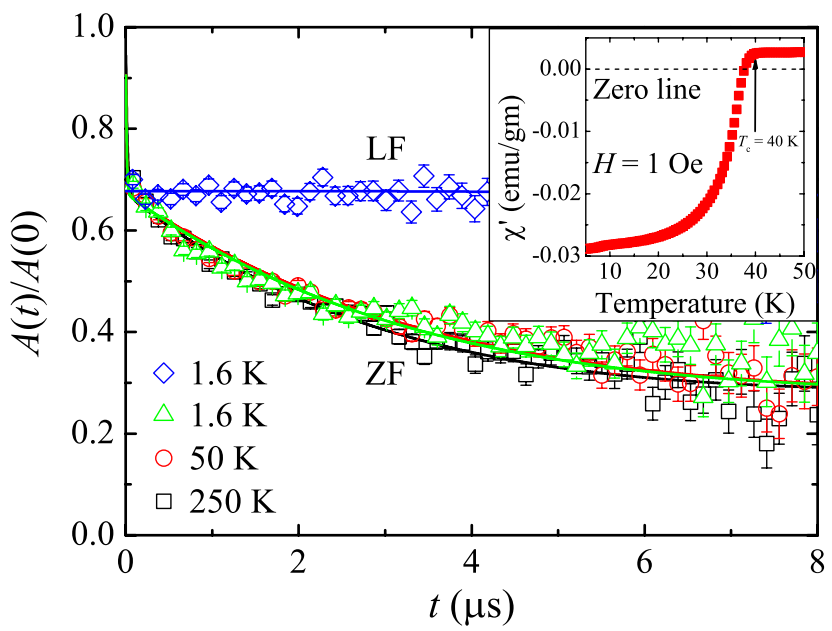

FIG. 1 (color online). ZF- $\mu \mathrm{SR}$ time spectra collected at $1.6 \mathrm{~K}$, $50 \mathrm{~K}$, and $250 \mathrm{~K}$ for $\mathrm{Li}\left(\mathrm{C}_{5} \mathrm{H}_{5} \mathrm{~N}\right)_{0.2} \mathrm{Fe}_{2} \mathrm{Se}_{2}$. LF- $\mu$ SR time spectra collected at $1.6 \mathrm{~K}$ and $200 \mathrm{Oe}$. The solid lines are the fits to the data using the equation described in the text. Inset shows the temperature dependence of the ac-magnetic susceptibility (real part only) of $\mathrm{Li}\left(\mathrm{C}_{5} \mathrm{H}_{5} \mathrm{~N}\right)_{0.2} \mathrm{Fe}_{2} \mathrm{Se}_{2}$ measured using the zero-fieldcooled mode under a magnetic field of $H_{\mathrm{ac}}=1 \mathrm{Oe}$.
ZF- $\mu$ SR signal, occurring within the first $100 \mathrm{ns,} \mathrm{over} \mathrm{the}$ entire temperature range. This suggests that some portion of our sample is purely magnetic. To calculate the volume fraction of the magnetic part, we have fitted the following equation to the ZF data:

$$
A^{\mathrm{ZF}}(t)=v_{m} A_{m}^{\mathrm{ZF}}(t)+\left(1-v_{m}\right) A_{n m}^{\mathrm{ZF}}(t),
$$

where

$$
\begin{aligned}
& A_{m}^{\mathrm{ZF}}(t)=A^{\mathrm{ZF}}(0)\left[\frac{2}{3} \exp \left(-\lambda_{T} t\right)+\frac{1}{3} \exp \left(-\lambda_{L} t\right)\right], \\
& A_{n m}^{\mathrm{ZF}}(t)=A^{\mathrm{ZF}}(0)\left[\frac{1}{3}+\frac{2}{3}\left(1-\sigma^{2} t^{2}-\Lambda t\right) \exp \left(-\frac{\sigma^{2} t^{2}}{2}-\Lambda t\right)\right] .
\end{aligned}
$$

Here $m$ and $\mathrm{nm}$ denote the magnetic and nonmagnetic components, $A^{\mathrm{ZF}}(0)$ is the initial asymmetry, $v_{m}$ is the magnetic volume fraction, and $\lambda_{T}, \lambda_{L}$ are the transverse and longitudinal components of the muon decay for the magnetic fraction of the sample, respectively. $A_{n m}^{\mathrm{ZF}}(t)$ is the combination of Lorentzian and Gaussian Kubo-Toyabe relaxation function [30,31] for the nonmagnetic fraction of the sample with parameters $\sigma$ and $\Lambda$, the muon depolarization rates arise due to the concentrated nuclear dipole moments and randomly orientated diluted local electronic moments, respectively. The fits yield the parameters shown in Table I. Parameters obtained from the fitting show that nearly half of the sample is antiferromagnetically ordered. This is similar to $\mathrm{Cs}, \mathrm{Rb}, \mathrm{K}$ intercalated FeSe superconductors [32]. The low relaxation rate $\Lambda$ most probably stems from the diluted ferromagnetic Fe impurity clusters. This hypothesis is supported by the fact that it is possible to decouple this slow relaxation with the application of a small longitudinal field (LF) of 200 Oe (see Fig. 1) which proves that the dipole field at the muon site is static on the time scale of the $\mu \mathrm{SR}$ window. The magnetic volume fraction is practically temperature independent between 1.6 and $250 \mathrm{~K}$. This strongly resembles the situation of phase separation in the alkali metals intercalated FeSe $[5,32]$. Our data show that there is a clear phase separation between the magnetic and the nonmagnetic fractions, and hence, the magnetic fraction is not affecting the properties of the nonmagnetic part of the sample.

We have also performed field shift measurements on $\mathrm{Li}\left(\mathrm{C}_{5} \mathrm{H}_{5} \mathrm{~N}\right)_{0.2} \mathrm{Fe}_{2} \mathrm{Se}_{2}$. The inset of Fig. 2(a) shows the internal field distribution, $B_{\text {int }}$ in the vortex state of

TABLE I. Parameters extracted from the fits using Eqs. (1) and (2) to the zero-field- $\mu \mathrm{SR}$ data collected above and below $T_{c}$ for $\mathrm{Li}\left(\mathrm{C}_{5} \mathrm{H}_{5} \mathrm{~N}\right)_{0.2} \mathrm{Fe}_{2} \mathrm{Se}_{2}$.

\begin{tabular}{lrc}
\hline \hline$T(\mathrm{~K})$ & \multicolumn{1}{c}{$v_{m}(\%)$} & $\Lambda$ \\
\hline 1.6 & $49.05 \pm 0.6$ & $0.18 \pm 0.01$ \\
50 & $50.8 \pm 0.6$ & $0.19 \pm 0.01$ \\
250 & $49.1 \pm 0.6$ & $0.20 \pm 0.01$ \\
\hline \hline
\end{tabular}


$\mathrm{Li}\left(\mathrm{C}_{5} \mathrm{H}_{5} \mathrm{~N}\right)_{0.2} \mathrm{Fe}_{2} \mathrm{Se}_{2}$ for the two different applied fields of 30 and 34 Oe. Field shift measurements show that an application of an additional 4 Oe magnetic field below $T_{c}$ does not shift the internal field distribution by a significant amount ( $\approx 0.5 \mathrm{G}$ only) due to vortex pinning. This shows that the paramagnetic volume fraction of the sample becomes fully superconducting below $T_{c}$.

To reveal the superconducting properties of $\mathrm{Li}\left(\mathrm{C}_{5} \mathrm{H}_{5} \mathrm{~N}\right)_{0.2} \mathrm{Fe}_{2} \mathrm{Se}_{2}$, we have performed a TF- $\mu$ SR study. It was very difficult to extract the superconducting properties of $\mathrm{Li}\left(\mathrm{C}_{5} \mathrm{H}_{5} \mathrm{~N}\right)_{0.2} \mathrm{Fe}_{2} \mathrm{Se}_{2}$ using a higher magnetic field due to higher relaxation from the diluted ferromagnetic impurities present in the sample. Therefore, we have used a small magnetic field of 30 Oe for this $\mathrm{TF}-\mu \mathrm{SR}$ study. It is noteworthy to mention that the lower critical field of all the iron chalcogenide superconductors are very low and in our case, 30 Oe was high enough to drive $\mathrm{Li}\left(\mathrm{C}_{5} \mathrm{H}_{5} \mathrm{~N}\right)_{0.2} \mathrm{Fe}_{2} \mathrm{Se}_{2}$ into a well-defined vortex state. Figure 2(a) shows the temperature dependence of the internal magnetic field at the muon sites in $\mathrm{Li}\left(\mathrm{C}_{5} \mathrm{H}_{5} \mathrm{~N}\right)_{0.2} \mathrm{Fe}_{2} \mathrm{Se}_{2}$. The dashed line is drawn as a guide

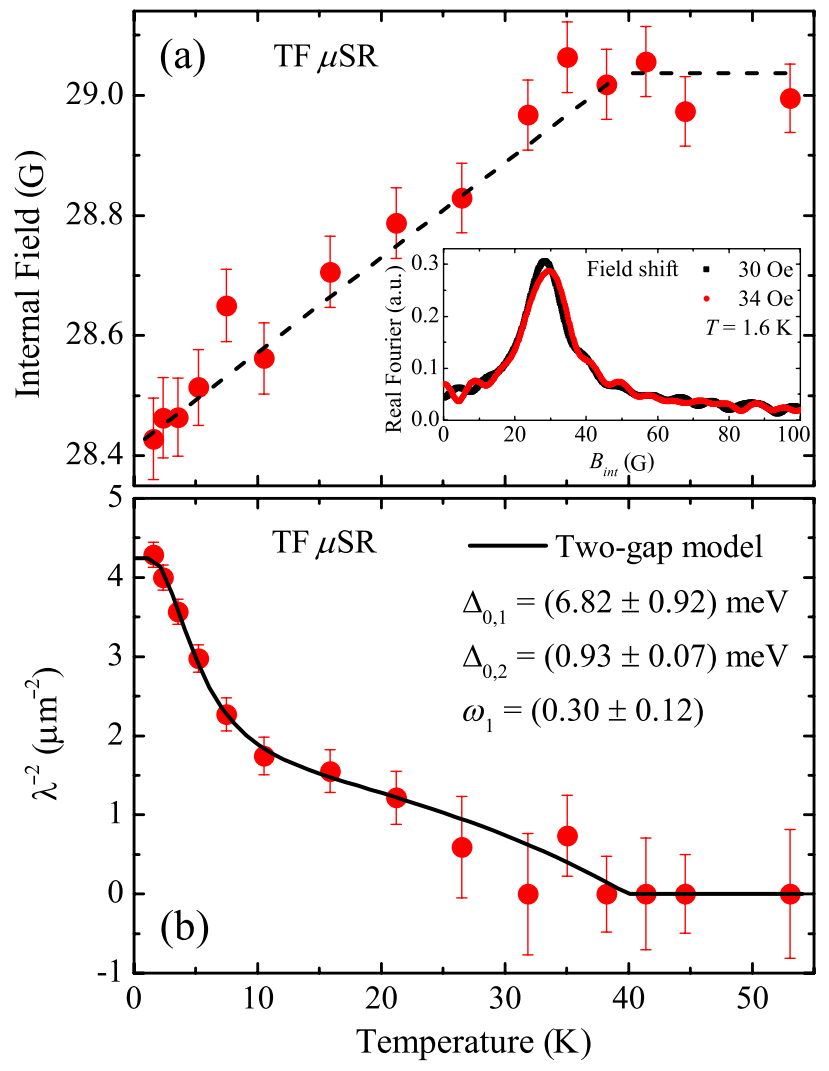

FIG. 2 (color online). Temperature dependence of the internal magnetic field at the muon sites in $\mathrm{Li}\left(\mathrm{C}_{5} \mathrm{H}_{5} \mathrm{~N}\right)_{0.2} \mathrm{Fe}_{2} \mathrm{Se}_{2}$. The dashed line is simply a guide to the eye. Inset shows the internal field distribution, $B_{\text {int }}$ of $\mathrm{Li}\left(\mathrm{C}_{5} \mathrm{H}_{5} \mathrm{~N}\right)_{0.2} \mathrm{Fe}_{2} \mathrm{Se}_{2}$ for the two different applied fields of 30 and 34 Oe. (b) Temperature dependence of $\lambda^{-2}$ for $\mathrm{Li}\left(\mathrm{C}_{5} \mathrm{H}_{5} \mathrm{~N}\right)_{0.2} \mathrm{Fe}_{2} \mathrm{Se}_{2}$. The curve (black line) is a fit to the data using two $s$-wave components, each with an isotropic gap. for the eye. We observed the expected diamagnetic shift of the internal magnetic field experienced by the muons below $T_{c}$.

To extract the superfluid density of $\operatorname{Li}\left(\mathrm{C}_{5} \mathrm{H}_{5} \mathrm{~N}\right)_{0.2} \mathrm{Fe}_{2} \mathrm{Se}_{2}$, we have fitted the TF muon time spectra using an oscillatory decaying Gaussian function,

$$
\begin{aligned}
A^{\mathrm{TF}}(t)= & A^{\mathrm{TF}}(0) \exp \left(-\sigma^{2} t^{2} / 2\right) \cos \left(\gamma_{\mu} B_{\mathrm{int}} t+\phi\right) \\
& +A_{\mathrm{bgd}}^{T F}(0) \cos \left(\gamma_{\mu} B_{\mathrm{bgd}} t+\phi\right),
\end{aligned}
$$

where $A^{\mathrm{TF}}(0)$ is the initial asymmetry, $\gamma_{\mu} / 2 \pi=$ $135.5 \mathrm{MHz} / \mathrm{T}$ is the muon gyromagnetic ratio [33], $B_{\text {int }}$ and $B_{\text {bgd }}$ are the internal and background magnetic field at the muon sites, $\phi$ is the initial phase offset of the muon precession signal, respectively, and $\sigma$ is the Gaussian muon spin relaxation rate. It can be written as $\sigma=\left(\sigma_{\mathrm{sc}}^{2}+\sigma_{n m}^{2}\right)^{1 / 2}$, where $\sigma_{\mathrm{sc}}$ is the superconducting contribution to the relaxation rate due to the field variation across the flux line lattice and $\sigma_{n m}$ is the nuclear magnetic dipolar contribution which is assumed to be constant over the temperature range of the study.

In a superconductor with a large upper critical field and a hexagonal Abrikosov vortex lattice, the Gaussian muonspin depolarization rate $\sigma_{\mathrm{sc}}$ is related to the penetration depth $\lambda$ by the expression

$$
\frac{\sigma_{\mathrm{sc}}^{2}(T)}{\gamma_{\mu}^{2}}=0.00371 \frac{\Phi_{0}^{2}}{\lambda^{4}(T)}
$$

where $\Phi_{0}=2.068 \times 10^{-15} \mathrm{~Wb}$ is the flux quantum [34]. Figure 2(b) shows the temperature dependence of $\lambda^{-2}$ for $\mathrm{Li}\left(\mathrm{C}_{5} \mathrm{H}_{5} \mathrm{~N}\right)_{0.2} \mathrm{Fe}_{2} \mathrm{Se}_{2}$.

The temperature dependence of the penetration depth of $\mathrm{Li}\left(\mathrm{C}_{5} \mathrm{H}_{5} \mathrm{~N}\right)_{0.2} \mathrm{Fe}_{2} \mathrm{Se}_{2}$ can be fitted using a two-gap $s$-wave model $[35,36]$,

$$
\frac{\lambda^{-2}(T)}{\lambda^{-2}(0)}=\omega \frac{\lambda^{-2}\left(T, \Delta_{1}\right)}{\lambda^{-2}\left(0, \Delta_{1}\right)}+(1-\omega) \frac{\lambda^{-2}\left(T, \Delta_{2}\right)}{\lambda^{-2}\left(0, \Delta_{2}\right)},
$$

where $\lambda(0)$ is the value of the penetration depth at $T=0 \mathrm{~K}, \Delta_{i}$ is the value of the $i$ th $(i=1$ or 2$)$ superconducting gap at $T=0 \mathrm{~K}$ and $\omega$ is the weighting factor of the first gap [37].

Each component in Eq. (5) can be expressed within the local London approximation [38,39] as

$$
\frac{\lambda^{-2}\left(T, \Delta_{i}\right)}{\lambda^{-2}\left(0, \Delta_{i}\right)}=1+2 \int_{\Delta_{i}}^{\infty}\left(\frac{\partial f}{\partial E}\right) \frac{E d E}{\sqrt{E^{2}-\Delta_{i}(T)^{2}}},
$$

where $f=\left[1+\exp \left(E / k_{B} T\right)\right]^{-1}$ is the Fermi function, and $\Delta_{i}(T)=\Delta_{i} \delta\left(T / T_{c}\right)$. The temperature dependence of the gap is approximated by the expression $\delta\left(T / T_{c}\right)=$ $\tanh \left\{1.82\left[1.018\left(T_{c} / T-1\right)\right]^{0.51}\right\}[35]$.

The curve shown in Fig. 2(b) is a fit of the two-gap $s$-wave model to the data. The fit yields $\Delta_{1}=$ 6.82(92) $\mathrm{meV}$ and $\Delta_{2}=0.93(7) \mathrm{meV}$ with $\omega=0.30(12)$. 
TABLE II. Superconducting gap to the $T_{c}$ ratios for different iron chalcogenide superconductors by means of $\mu$ SR study.

\begin{tabular}{llcc}
\hline \hline Compounds & $2 \Delta_{1} / k_{B} T_{c}$ & $2 \Delta_{2} / k_{B} T_{c}$ & References \\
\hline $\mathrm{FeSe}_{0.85}$ & $4.49(6)$ & $1.07(2)$ & {$[25]$} \\
$\mathrm{FeTe}_{0.5} \mathrm{Se}_{0.5}$ & $4.19(16)$ & $1.40(9)$ & {$[40]$} \\
$\mathrm{K}_{0.74} \mathrm{Fe}_{1.66} \mathrm{Se}_{2}$ & $4.7(2)$ & & {$[32]$} \\
$\mathrm{Rb}_{0.77} \mathrm{Fe}_{1.61} \mathrm{Se}_{2}$ & $5.5(2)$ & & {$[32]$} \\
$\mathrm{Li}_{0.6}\left(\mathrm{NH}_{2}\right)_{0.2}\left(\mathrm{NH}_{3}\right)_{0.8} \mathrm{Fe}_{2} \mathrm{Se}_{2}$ & $5.27(21)$ & $0.69(2)$ & {$[21]$} \\
$\mathrm{Li}\left(\mathrm{C}_{5} \mathrm{H}_{5} \mathrm{~N}\right)_{0.2} \mathrm{Fe}_{2} \mathrm{Se}_{2}$ & $3.96(54)$ & $0.57(4)$ & Current \\
\hline \hline
\end{tabular}

The ratio of the larger to the smaller gap, $\Delta_{1} / \Delta_{2} \sim 7.3(2)$ found in $\mathrm{Li}\left(\mathrm{C}_{5} \mathrm{H}_{5} \mathrm{~N}\right)_{0.2} \mathrm{Fe}_{2} \mathrm{Se}_{2}$ is larger than the corresponding value (4.2) seen in $\mathrm{FeSe}_{0.85}$ but is consistent with the value (7.7) for the lithium amide and ammonia intercalated FeSe determined by measuring the magnetic penetration depth using the $\mu$ SR technique $[21,25]$. The large gap to the $T_{c}$ ratio is $2 \Delta_{1} / k_{B} T_{c}=3.96(54)$ and the small gap to the $T_{c}$ ratio is $2 \Delta_{2} / k_{B} T_{c}=0.57(4)$. This shows that the large gap is in the strong-coupling limit whereas the small gap is in the weak-coupling limit. Table II summarizes the superconducting gap to the $T_{c}$ ratios for different iron chalcogenide superconductors by means of $\mu$ SR study.

The value of the penetration depth at $T=0 \mathrm{~K}$ is found to be $\lambda(0)=485(21) \mathrm{nm}$ for $\mathrm{Li}\left(\mathrm{C}_{5} \mathrm{H}_{5} \mathrm{~N}\right)_{0.2} \mathrm{Fe}_{2} \mathrm{Se}_{2}$. For a $\mathrm{n}$ anisotropic polycrystalline sample, $\lambda$ is related to $\lambda_{a b}$ by $\lambda=3^{1 / 4} \lambda_{a b}$ [41]. With this assumption, we found $\lambda_{a b}(0)=369(16) \mathrm{nm}$ for $\mathrm{Li}\left(\mathrm{C}_{5} \mathrm{H}_{5} \mathrm{~N}\right)_{0.2} \mathrm{Fe}_{2} \mathrm{Se}_{2}$. These values are comparable with those obtained by Khasanov et al. [25] for $\mathrm{FeSe}_{0.85}$ but $50 \%$ higher than the value obtained from $\mathrm{Li}_{0.6}\left(\mathrm{NH}_{2}\right)_{0.2}\left(\mathrm{NH}_{3}\right)_{0.8} \mathrm{Fe}_{2} \mathrm{Se}_{2}$ by Lucas et al. [21] using $\mu \mathrm{SR}$. This discrepancy may be due to the fact that the interlayer spacing between the FeSe layers in our compound (lattice parameter, $c=23.09648 \AA$ ) is $40 \%$ higher than $\mathrm{Li}_{0.6}\left(\mathrm{NH}_{2}\right)_{0.2}\left(\mathrm{NH}_{3}\right)_{0.8} \mathrm{Fe}_{2} \mathrm{Se}_{2}(c=16.5266 \AA)$. Recent $\mu$ SR studies on bismuth-based high- $T_{c}$ cuprate superconductors by Baker et al. [42] suggest that the penetration depth in that class of materials increases with increasing layer separation between the $\mathrm{CuO}_{2}$ layers with a relation $1 / d \propto 1 / \lambda_{a b}^{2}$, where $d$ is the interlayer distance. It is also found that the $T_{c}$ value depends only on the $2 \mathrm{D}$ superfluid density, but not on the bulk superfluid density. A modified Uemura plot for the 2D superfluid density of the iron chalcogenide superconductors is shown in Fig. 3. The value for $\lambda_{a b}^{2}$ place $\mathrm{Li}\left(\mathrm{C}_{5} \mathrm{H}_{5} \mathrm{~N}\right)_{0.2} \mathrm{Fe}_{2} \mathrm{Se}_{2}$ well inside the broad line for 2D superfluid densities of iron chalcogenide superconductors on an Uemura plot. This suggests that the superfluid density of $\mathrm{Li}\left(\mathrm{C}_{5} \mathrm{H}_{5} \mathrm{~N}\right)_{0.2} \mathrm{Fe}_{2} \mathrm{Se}_{2}$ is $2 \mathrm{D}$ in nature and that the superfluid densities appearing in the Uemura scaling relation should be modified as 2D for these Fe-based superconductors. This also points to a noteworthy similarity between the Fe-based and high- $T_{c}$ cuprate superconductors. In analogy with the $\mathrm{CuO}_{2}$ layer in the high- $T_{c}$ cuprates, we then suggest that it is the $2 \mathrm{D} \mathrm{FeSe}$ layer which generates superconductivity in this system.

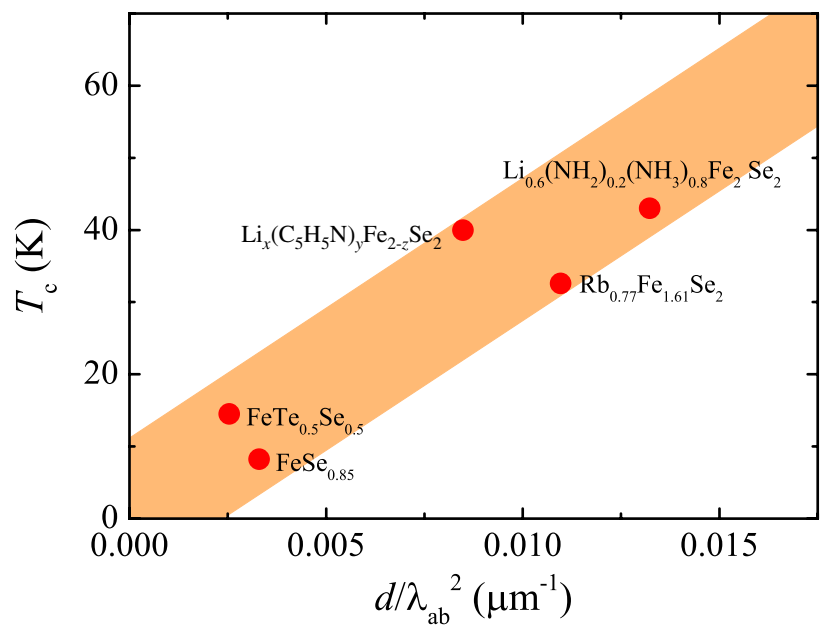

FIG. 3 (color online). Uemura plot of $T_{c}$ vs $d / \lambda_{a b}^{2}$ for $2 \mathrm{D}$ superfluid density of iron chalcogenide superconductors.

In summary, $\mu \mathrm{SR}$ measurements have been performed on the alkali metal-organic solvent intercalated iron selenide superconductor $\mathrm{Li}\left(\mathrm{C}_{5} \mathrm{H}_{5} \mathrm{~N}\right)_{0.2} \mathrm{Fe}_{2} \mathrm{Se}_{2}$ with a $T_{c}$ of 40(1) $\mathrm{K}$. The superfluid density of this compound is found to be very low compared to other iron-chalcogenide superconductors, probably due to its 2D nature. Nearly $50 \%$ (volume fraction) of the sample is magnetically ordered. A spatial phase separation has been observed between the superconducting and the magnetic fraction of the sample. The temperature dependence of the magnetic penetration depth is found to be compatible with a two-gap $s$-wave model with gap values $\Delta_{1}=6.82(92) \mathrm{meV}$ and $\Delta_{2}=$ 0.93(7) meV. We obtain $\lambda(0)=485(21) \mathrm{nm}$ at $T=0 \mathrm{~K}$. Further studies are in progress to explore the magnetic properties and whether there is any microscopic coexistence of superconductivity and magnetism in the magnetic volume of this material.

The $\mu \mathrm{SR}$ experiments were performed at the Swiss Muon Source, Paul Scherrer Institut, Villigen, Switzerland. A. K.-M. gratefully acknowledges the financial support from Institute of Physics University of Zürich. The $\mu$ SR data were analyzed using the free software package MUSRFIT [43].

*pabitra.biswas@psi.ch

[1] Y. Kamihara, T. Watanabe, M. Hirano, and H. Hosono, J. Am. Chem. Soc. 130, 3296 (2008).

[2] C. Wang, L. Li, S. Chi, Z. Zhu, Z. Ren, Y. Li, Y. Wang, X. Lin, Y. Luo, S. Jiang et al., Europhys. Lett. 83, 67006 (2008).

[3] J. Guo, S. Jin, G. Wang, S. Wang, K. Zhu, T. Zhou, M. He, and X. Chen, Phys. Rev. B 82, 180520 (2010).

[4] A. Krzton-Maziopa, Z. Shermadini, E. Pomjakushina, V. Pomjakushin, M. Bendele, A. Amato, R. Khasanov, H. Luetkens, and K. Conder, J. Phys. Condens. Matter 23, 052203 (2011). 
[5] Z. Shermadini, A. Krzton-Maziopa, M. Bendele, R. Khasanov, H. Luetkens, K. Conder, E. Pomjakushina, S. Weyeneth, V. Pomjakushin, O. Bossen et al., Phys. Rev. Lett. 106, 117602 (2011).

[6] D. A. Torchetti, M. Fu, D. C. Christensen, K. J. Nelson, T. Imai, H. C. Lei, and C. Petrovic, Phys. Rev. B 83, 104508 (2011).

[7] W. Yu, L. Ma, J. B. He, D. M. Wang, T.-L. Xia, G. F. Chen, and W. Bao, Phys. Rev. Lett. 106, 197001 (2011).

[8] L. Ma, G. F. Ji, J. Zhang, J. B. He, D. M. Wang, G. F. Chen, W. Bao, and W. Yu, Phys. Rev. B 83, 174510 (2011).

[9] H. Kotegawa, Y. Hara, H. Nohara, H. Tou, Y. Mizuguchi, H. Takeya, and Y. Takano, J. Phys. Soc. Jpn. 80, 043708 (2011).

[10] F.-C. Hsu, J.-Y. Luo, K.-W. Yeh, T.-K. Chen, T.-W. Huang, P. M. Wu, Y.-C. Lee, Y.-L. Huang, Y.-Y. Chu, D.-C. Yan et al., Proc. Natl. Acad. Sci. U.S.A. 105, 14262 (2008).

[11] S. Margadonna, Y. Takabayashi, Y. Ohishi, Y. Mizuguchi, Y. Takano, T. Kagayama, T. Nakagawa, M. Takata, and K. Prassides, Phys. Rev. B 80, 064506 (2009).

[12] Q.-Y. Wang, Z. Li, W.-H. Zhang, Z.-C. Zhang, J.-S. Zhang, W. Li, H. Ding, Y.-B. Ou, P. Deng, K. Chang et al., Chin. Phys. Lett. 29, 037402 (2012).

[13] D. Liu, W. Zhang, D. Mou, J. He, Y.-B. Ou, Q.-Y. Wang, Z. Li, L. Wang, L. Zhao, S. He et al., Nat. Commun. 3, 931 (2012)

[14] S. He, J. He, W. Zhang, L. Zhao, D. Liu, X. Liu, D. Mou, Y.-B. Ou, Q.-Y. Wang, Z. Li et al., arXiv:1207.6823v1.

[15] K.-W. Yeh, T.-W. Huang, Y.-1. Huang, T.-K. Chen, F.-C. Hsu, P. M. Wu, Y.-C. Lee, Y.-Y. Chu, C.-L. Chen, J.-Y. Luo et al., Europhys. Lett. 84, 37002 (2008).

[16] M. H. Fang, H. M. Pham, B. Qian, T. J. Liu, E. K. Vehstedt, Y. Liu, L. Spinu, and Z. Q. Mao, Phys. Rev. B 78, 224503 (2008).

[17] N.C. Gresty, Y. Takabayashi, A. Y. Ganin, M. T. McDonald, J. B. Claridge, D. Giap, Y. Mizuguchi, Y. Takano, T. Kagayama, Y. Ohishi et al., J. Am. Chem. Soc. 131, 16944 (2009).

[18] A. F. Wang, J. J. Ying, Y. J. Yan, R. H. Liu, X. G. Luo, Z. Y. Li, X. F. Wang, M. Zhang, G. J. Ye, P. Cheng et al., Phys. Rev. B 83, 060512 (2011).

[19] L. Sun, X.-J. Chen, J. Guo, P. Gao, H. Wang, M. Fang, X. Chen, G. Chen, Q. Wu, C. Zhang et al., Nature (London) 483, 67 (2012).

[20] T. P. Ying, X. L. Chen, G. Wang, S. F. Jin, T. T. Zhou, X. F. Lai, H. Zhang, and W. Y. Wang, Sci. Rep. 2, 426 (2012).

[21] M. Burrard-Lucas, D. G. Free, S. J. Sedlmaier, J. D. Wright, S. J. Cassidy, Y. Hara, A. J. Corkett, T. Lancaster, P. J. Baker, S. J. Blundell et al., Nat. Mater. 12, 15 (2012).

[22] E.-W. Scheidt, V. R. Hathwar, D. Schmitz, A. Dunbar, W. Scherer, V. Tsurkan, J. Deisenhofer, and A. Loidl, Eur. Phys. J. B 85, 279 (2012).
[23] A. Krzton-Maziopa, E. Pomjakushina, V. Y. Pomjakushin, F. von Rohr, A. Schilling, and K. Conder, J. Phys. Condens. Matter 24, 382202 (2012).

[24] A. M. Zhang, T. L. Xia, K. Liu, W. Tong, Z. R. Yang, and Q. M. Zhang, Sci. Rep. 3, 1216 (2013).

[25] R. Khasanov, K. Conder, E. Pomjakushina, A. Amato, C. Baines, Z. Bukowski, J. Karpinski, S. Katrych, H.-H. Klauss, H. Luetkens et al., Phys. Rev. B 78, 220510 (2008).

[26] S. B. Zhang, X. D. Zhu, H.C. Lei, G. Li, B.S. Wang, L. J. Li, X. B. Zhu, Z. R. Yang, W. H. Song, J. M. Dai et al., Supercond. Sci. Technol. 22, 075016 (2009).

[27] U. Patel, J. Hua, S. H. Yu, S. Avci, Z. L. Xiao, H. Claus, J. Schlueter, V. V. Vlasko-Vlasov, U. Welp, and W. K. Kwok, Appl. Phys. Lett. 94, 082508 (2009).

[28] T. M. McQueen, Q. Huang, V. Ksenofontov, C. Felser, Q. Xu, H. Zandbergen, Y. S. Hor, J. Allred, A. J. Williams, D. Qu et al., Phys. Rev. B 79, 014522 (2009).

[29] R. Hu, H. Lei, M. Abeykoon, E. S. Bozin, S. J. L. Billinge, J. B. Warren, T. Siegrist, and C. Petrovic, Phys. Rev. B 83, 224502 (2011).

[30] R. Kubo and T. Toyabe, Magnetic Resonance and Relaxation (North Holland, Amsterdam, 1967).

[31] R. S. Hayano, Y. J. Uemura, J. Imazato, N. Nishida, T. Yamazaki, and R. Kubo, Phys. Rev. B 20, 850 (1979).

[32] Z. Shermadini, H. Luetkens, R. Khasanov, A. KrztonMaziopa, K. Conder, E. Pomjakushina, H.-H. Klauss, and A. Amato, Phys. Rev. B 85, 100501 (2012).

[33] J. E. Sonier, J. H. Brewer, and R. F. Kiefl, Rev. Mod. Phys. 72, 769 (2000).

[34] E. H. Brandt, Phys. Rev. B 37, 2349 (1988).

[35] A. Carrington and F. Manzano, Physica (Amsterdam) 385, 205 (2003).

[36] H. Padamsee, J. E. Neighbor, and C. A. Shiffman, J. Low Temp. Phys. 12, 387 (1973).

[37] Note that the $\mu$ SR technique can not determine the phase of the superconducting order parameter and therefore can not distinguish between $s^{+}$and $s^{-}$-wave gaps. For the two-gap $s$-wave model, we have simply added two single $s$-wave gap equations along with a weighting factor.

[38] M. Tinkham, Introduction to Superconductivity (McGrawHill, New York, 1975).

[39] R. Prozorov and R.W. Giannetta, Supercond. Sci. Technol. 19, R41 (2006).

[40] P. K. Biswas, G. Balakrishnan, D. M. Paul, C. V. Tomy, M. R. Lees, and A. D. Hillier, Phys. Rev. B 81, 092510 (2010).

[41] V. I. Fesenko, V. N. Gorbunov, and V. P. Smilga, Physica (Amsterdam) 176, 551 (1991).

[42] P. J. Baker, T. Lancaster, S. J. Blundell, F. L. Pratt, M. L. Brooks, and S.-J. Kwon, Phys. Rev. Lett. 102, 087002 (2009).

[43] A. Suter and B. M. Wojek, Phys. Procedia 30, 69 (2012). 